\title{
Enhanced Intracellular Uptake of CdTe Quantum Dots by Conjugation of Oligopeptides
}

\author{
Moon-Jeong Choi, ${ }^{1}$ R. Pierson, ${ }^{1}$ Yongmin Chang, ${ }^{2}$ Haiquing Guo, ${ }^{3}$ and Inn-Kyu Kang ${ }^{1}$ \\ ${ }^{1}$ Department of Polymer Science and Engineering, Kyungpook National University, Daegu 702-701, Republic of Korea \\ ${ }^{2}$ Department of Diagnostic Radiology, Kyungpook National University, Daegu 702-701, Republic of Korea \\ ${ }^{3}$ College of Chemistry and Molecular Engineering, Peking University, Beijing 100-871, China \\ Correspondence should be addressed to Inn-Kyu Kang; ikkang@knu.ac.kr
}

Received 10 January 2013; Accepted 3 April 2013

Academic Editor: Miguel A. Correa-Duarte

Copyright (C) 2013 Moon-Jeong Choi et al. This is an open access article distributed under the Creative Commons Attribution License, which permits unrestricted use, distribution, and reproduction in any medium, provided the original work is properly cited.

Arg-Gly-Asp-Ser (RGDS), a typical membrane-permeable carrier peptide, was conjugated with mercaptoisobutyric acid-immobilized CdTe quantum dot (CTNPs) to enhance the intracellular uptake of quantum dots. Mean size of mercaptoisobutyric acidimmobilized quantum dots $(37 \mathrm{~nm})$ as determined by dynamic light scattering was increased up to $54 \mathrm{~nm}$ after RGDS immobilization. It was found, from in vitro cell culture experiment, that fibroblast (NIH 3T3) cells were well proliferated in the presence of RGDS-conjugated quantum dots (RCTNPs), and the intracellular uptake of CTNPs and RCTNPs was studied by means of ICP and fluorescence microscopy. As a result, the RCTNPs specifically bound to the membrane of NIH 3T3 cells and almost saturated after 6 hours incubation. The amount of RCTNPs uptaken by the cells was higher than that of CTNPs, demonstrating the enhancing effect of RGDS peptide conjugation on the intracellular uptake of quantum dots (QDs).

\section{Introduction}

The development of noncytotoxic quantum dots (QDs) has attracted tremendous interest as luminescence probs in biological and medical research due to some of their unique optical and chemical properties [1], such as tunable fluorescence wavelength by size, a sharp and symmetrical fluorescence peak, strong and stable emission, high quantum yield, brightness, and photo stability [2-5]. Compared with the traditional dyes and fluorescent proteins used as imaging probes, QDs have several advantages, such as tunable emission from visible to infrared wavelengths, broader excitation spectra, and high resistance to photobleaching [6, 7]. However, the potential applications of QDs in biology and medicine were limited due to their toxic effects [8]. QDs contain toxic components such as cadmium or lead. $\mathrm{Cd}^{2+}$ and $\mathrm{Pb}^{2+}$ could be released from QDs and then kill the cells [9]. Recently, a number of techniques such as a gold outer shell [10], targeted ligand-like peptide [3], proteins [11, 12], and polymer coating [13] have been developed to minimize the cytotoxicity of QDs. Consequently, many approaches for transforming
QDs from hydrophobic to hydrophilic have been developed for various biomedical applications. Therefore, alternative researchers have also used noncytotoxic materials such as polyethylene glycol (PEG) and polymaleic anhydride salt-1tetradecene to coat the surfaces of QDs [14]. Depending on the applications, surface modifications of QDs are required. For example, biomedical applications require high-quality water soluble and nontoxic QDs.

So far, numerous modifications of QDs surface chemistry have been explored, including the attachment of mercaptoacetic acid [15], mercaptopropionic acid [16], mercaptobenzoic acid [17], and biocompatible and chemically functionalizable inorganic shells such as silica or zinc sulfide [18]. All these coatings can mainly ensure the water solubility of QDs and are unable to enhance the biocompatibility considerably. Therefore, further coating with suitable watersoluble organic ligand/biomolecules is necessary to enhance the biocompatibility of QDs. To that end, so far QDs have been covalently linked with biorecognition molecules such as biotin [19], folic acid [20], peptides [21], bovine serum albumin [14], transferrin [22], antibodies [23], and DNA [24]. 
In this study, mercaptoisobutyric acid (MIBA)-coated cadmium telluride quantum dots (CTNPs) nanoparticles were prepared. Then, the arginine-glycine-asparagine-serine (RGDS) tetrapeptide was immobilized on the MIBA modified CdTe quantum dots (RCTNPs). Arg-Gly-Asp (RGD) peptide, an integrin recognition motif found in fibronectin and one of the most extensively studied cell adhesion peptides [25], was incorporated into stabilization of quantum dots (RCTNPs). RGDS is short heterobifunctional peptides for surface functionalization and stabilization of QDs. Peptides are small but carry the inherent advantage of easy customization for specific applications. RGDS contains two different functional moieties, the side-chain thiol(s) of cysteine (Cys) residue(s) to bind to the QDs surface and control the surface passivation and the RGD sequence (arginine-glycine-aspartic acid) pointing toward the surrounding aqueous solution and mediating the exemplary biorecognition [26]. CTNPs and RCTNPs were then characterized by Fourier-transform infrared (FT-IR) spectrophotometer, field emission transmission electron microscope (FE-TEM), dynamic light scattering (DLS), and zeta potential. Interaction of CTNPs and RCTNPs with fibroblast cells (NIH 3T3) were investigated by examining intracellular uptake of the particles into target cells using inductively coupled plasma (ICP) and fluorescence microscope.

\section{Experimental}

2.1. Material. Cadmium chloride $\left(\mathrm{CdCl}_{2}\right)$, sodium tellurite $\left(\mathrm{Na}_{2} \mathrm{TeO}_{3}\right)$, mercaptoisobutyric acid (MIBA), and surfactants as 1-ethyl-3-(3-dimethylaminopropyl)-carbodiimide (EDC) and N-hydroxy succinimide were purchased from SigmaAldrich Co. (USA). Cell culture reagents, fetal bovine serum (FBS), Dulbecco's Modified Eagle Medium (DMEM, high glucose), penicillin-streptomycine, trypsin/EDTA, Dulbecco's phosphate buffer saline (PBS), and promokine livedead cell staining kit were purchased from Gibco BRL (Carlsbad, CA), and NIH 3T3 cells (animal fibroblast cells) were purchased from Korean Cell Line Bank.

2.2. Synthesis of Water Soluble CTNPs. Water soluble CTNPs were synthesized by previously published method [15]. Briefly, carboxyl-stabilized CTNPs were synthesized by arrested precipitation at room temperature in an aqueous solution using MIBA as the colloidal stabilizer. Nanocrystals were prepared from a stirred solution of $0.0456 \mathrm{~g} \mathrm{CdCl}_{2}$ $(5 \mathrm{mM})$ in $40 \mathrm{~mL}$ of pure water. The $\mathrm{pH}$ was lowered to 2 with MIBA and then raised to 9 with $3 \mathrm{~N} \mathrm{NaOH}$. The mixture was deaerated by $\mathrm{N}_{2}$ bubbling for about $30 \mathrm{~min}$, and then, a freshly prepared $40 \mathrm{~mL}$ of $5 \mathrm{mM}$ sodium tellurite $\left(\mathrm{Na}_{2} \mathrm{TeO}_{3}\right)$ was added to the mixture with rapid stirring. The solution turned yellow shortly after the formation of cadmium tellurite nanoparticles (CTNPs) (reaction scheme shown in Figure 1). CTNPs were separated from the reaction byproducts by precipitation with the addition of acetone $(4 \mathrm{~mL}$ of acetone $/ \mathrm{mL}$ of nanocrystal solution). The precipitate was then isolated by centrifugation and dried in a freeze dryer. The precipitate powder CTNPs are redispersible in water, whereby a clear colloidal solution is obtained. The free carboxylic acid groups of the prepared CTNPs are suitable for covalent coupling to various biomolecules by covalent interaction of reactive amine groups.

2.3. Immobilization of RGDS Peptide on CTNPs. Immobilization of RGDS peptide on CTNPs (Figure 2) involves two steps; first, CTNPs were reacted with ethylenediamine to introduce amine group on the surface. To do so, CTNPs $(0.3 \mathrm{~g})$ were dissolved in an aqueous solution $(20 \mathrm{~mL})$ containing 1-ethyl-3-(3-dimethyl-aminopropyl)-carbodiimide hydrochloride (EDC, $4 \mathrm{mg} / \mathrm{mL}$ ) and stirred for 4 hours to activate the carboxyl groups on the surfaces. In the second step, the RGDS peptide was immobilized on the surface of CTNPs as follows: RGDS peptide $(0.3 \mathrm{~g})$ was dissolved in a phosphate buffer solution ( $40 \mathrm{~mL}, \mathrm{pH} 7)$ containing 1-ethyl3-(3-dimethyl-aminopropyl)-carbodiimide hydrochloride (EDC) and stirred for 5 hours to activate the carboxylic acid groups of RGDS peptide. Then, CTNPs $(0.3 \mathrm{~g})$ were added to this solution, and mixture was stirred for 48 hours at room temperature to obtain RGDS peptide-coated CTNPs (RCTNPs) (reaction scheme is shown in Figure 2). RCTNPs were isolated by repeated centrifugation and stored in phosphate buffered saline (PBS) at $\mathrm{pH}$ 7. All the conjugation reactions, unless otherwise noted, were carried out in a dark under an $\mathrm{N}_{2}$ ambient environment.

2.4. Surface Characterization. Quantum dots nanoparticles were ground with $\mathrm{KBr}$ powder, compressed into pellets, and subjected to the measurement of Fourier transform infrared (Jasco FTIR 300 E spectrometer) with a resolution of $4 \mathrm{~cm}^{-1}$. Transmission electron microphotographs (Philips, CM 200 TEM, applied operation voltage; $120 \mathrm{kV}$ ) were used to observe the morphology of nanoparticles. To obtain the samples for TEM observations, particles were diluted with distilled water and then deposited on Formvar-coated 400 mesh copper grids. After drying the nanoparticles fluids thin films on the copper grid, a thin layer of carbon films of about 10$30 \mathrm{~nm}$ in thickness was deposited on the nanoparticles fluids film. The hydrodynamic diameter and size distribution of the quantum dots was determined by dynamic light scattering (DLS) by means of a standard laboratory built light scattering spectrometer using a BI90 particle sizer (Brookhaven Instruments Corp., Holtsville, NY). It had a vertically polarized incident light of $514.5 \mathrm{~nm}$ supplied by an argon ion laser (Lexel laser, model 95). The UV-vis absorption spectrum was recorded from aqueous dispersions at room temperature using a Hitachi U-3000 spectrophotometer.

2.5. Cell Culture. Animal fibroblast cells (NIH 3T3) were used in this experiment. Cells were routinely cultured at $37^{\circ} \mathrm{C}$ in a humidified atmosphere with $5 \% \mathrm{CO}_{2}$ (in air), in a $75 \mathrm{~cm}^{2}$ flask containing $10 \mathrm{~mL}$ of DMEM, supplemented with $10 \%$ fetal bovine serum and $1 \%$ penicillin streptomycin $\mathrm{G}$ sodium (PGS). Medium was changed every three days. For subculture, the cells were washed twice with PBS and incubated with trypsin-EDTA solution ( $0.25 \%$ trypsin, $1 \mathrm{mM}$ EDTA) for $10 \mathrm{~min}$ at $37^{\circ} \mathrm{C}$ to detach the cells, and complete media were then added to the flask at room temperature to inhibit the effect of trypsin. The cells were washed twice by 


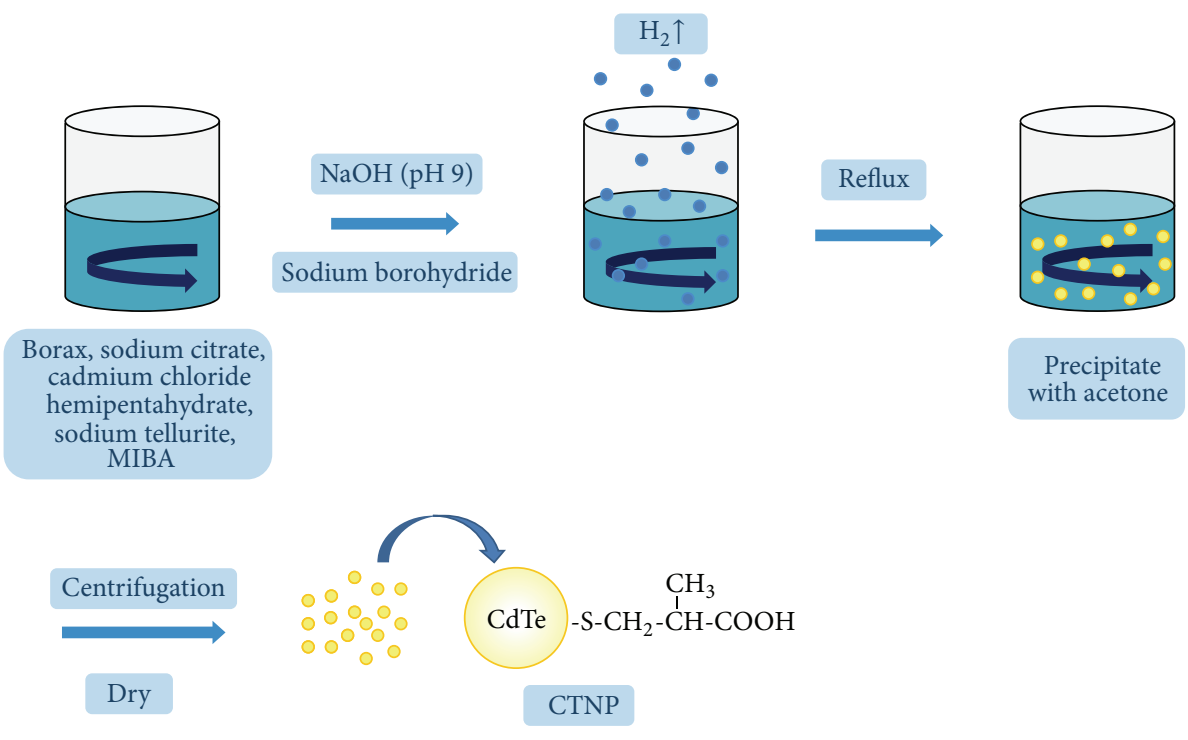

FIGURE 1: Schematic diagram showing synthesis of mercaptoisobutyric acid (MIBA)-coated CdTe nanoparticle (CTNP).

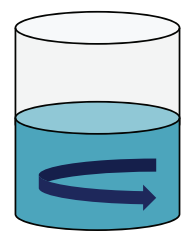

CTNP
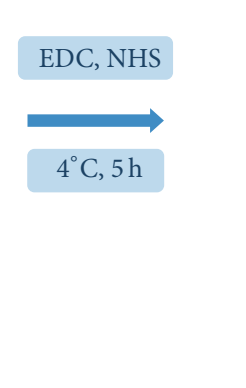

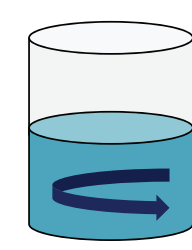

$$
\begin{gathered}
\text { RGDS peptide } \\
+ \\
\text { activated CTNP }
\end{gathered}
$$

RCTNP

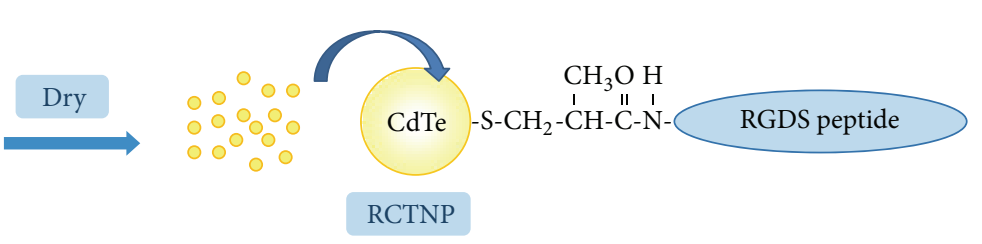

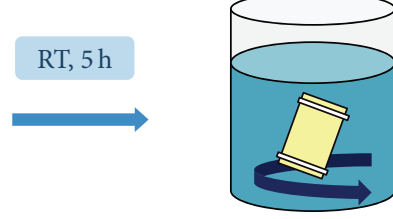

Dialysis

FIGURE 2: Schematic diagram showing immobilization of RGDS on CTNP (RCTNP).

centrifugation and resuspended in the complete media for reseeding and growing in new culture flasks. Cell viability was determined through staining with trypan blue, and cells were counted using a hemocytometer. Cell density was estimated using a $0.9 \mathrm{~mm}^{3}$ counting chamber.

2.6. Intracellular Uptake of CTNPs. To study the cellular uptake of the nanoparticles via fluorescence and confocal laser microscopy, CTNPs and RCTNPs were added to the cell culture medium at a particle concentration of $100 \mu \mathrm{g} / \mathrm{mL}$. The cells were cultured in 24-well plastic dishes for 20 hours and then reseeded with the nano particle-dispersed culture medium. After 48 hours of incubation at $37^{\circ} \mathrm{C}$, the cells were washed twice with PBS, detached using trypsin-EDTA solution, and resuspended in $10 \mathrm{~mL}$ culture media, and $1 \mathrm{~mL}$ cell suspension was reseeded on $18-\mathrm{mm}$ coverslips placed in a new 24-well plastic dish. After an additional 24 hours of culture, the dishes were washed with PBS and fixed with $4 \%$ paraformaldehyde solution at $4^{\circ} \mathrm{C}$ for $15 \mathrm{~min}$ followed by washing with deionized water, and the coverslips were put on the slide coated with buffered mounting medium to prevent fading.

To quantify intracellular uptake of the nanoparticles, cells were grown in 24-well culture plates with approximately $10^{5}$ cells in $1 \mathrm{~mL}$ of the medium. After incubation at $37^{\circ} \mathrm{C}$ for 20 hours, the cells were reseeded with a culture medium containing quantum dot nanoparticles at a concentration of $2 \times 10^{-4}$ cell $/ \mathrm{mL}$. In control cultures, the cells were placed in $1 \mathrm{~mL}$ medium without quantum dot nanoparticles at the same cell density. The intracellular cadmium concentration was quantified using inductively coupled plasma (ICP). Cells were washed with PBS, detached, resuspended, counted, and centrifuged down, and the cell pellets were dissolved in $37 \% \mathrm{HCl}$ aqueous solution at $70-80^{\circ} \mathrm{C}$ for $30 \mathrm{~min}$. The 
samples were diluted to a final cadmium concentration of 1.0$4.0 \mu \mathrm{g} / \mathrm{mL}$. The experiment was repeated three times, and the results were averaged with standard deviation.

2.7. Cell Viability. The proliferation of NIH $3 \mathrm{~T} 3$ cells in a medium containing CTNPs and RCTNPs as determined using the MTT (3-\{4,5-dimethylthiazol-2yl\}-2,5-diphenyltetrazolium bromide) assay. Briefly, NIH3T3 cells were seeded $\left(1 \times 10^{5} \mathrm{cell} / \mathrm{mL}\right)$ on 24 -well plates in the presence of a cell culture medium. After $24 \mathrm{~h}$, the culture medium was replaced with a fresh medium containing CTNPs and RCTNPs at a particle concentration of $0.2 \mathrm{mg} / \mathrm{mL}$. After being incubated for 1,2 , and 3 days, a $50 \mu \mathrm{L}$ MTT solution ( $5 \mathrm{mg} / \mathrm{mL}$ in PBS) was added to each well and incubated in a humidified atmosphere of $5 \% \mathrm{CO}_{2}$ at $37^{\circ} \mathrm{C}$ for $4 \mathrm{~h}$. After removing the medium, the converted dye was dissolved in acidic isopropanol $(0.04 \mathrm{~N}$ $\mathrm{HCl}$-isopropanol) and kept for $30 \mathrm{~min}$ in the dark at room temperature. From each sample, the medium $(100 \mu \mathrm{L})$ was taken and transferred to a 96-well plate and subjected to ultraviolet measurements for converted dye. This was done at a wavelength of $570 \mathrm{~nm}$ on a kinetic microplate reader, and the experiment was repeated three times. The fluorescence images of live-dead cells containing the nanoparticles were obtained by adding $5 \mu \mathrm{L}$ of Promokine live-dead cell staining solutions to the culture plates after the removal of medium and incubated in a humidified atmosphere of $5 \% \mathrm{CO}_{2}$ at $37^{\circ} \mathrm{C}$ for $15 \mathrm{~min}$.

2.8. Fluorescence Microscopic Images. The phase contrast and fluorescence images of cells were obtained with a combined explorer system with a motorized inverted fluorescence microscope (Carl Zeiss LSM700 Germany), using the topographic images which can be detected simultaneously.

2.9. Statistical Analysis. The cell proliferation experiment was performed in triplicate, and the results were expressed as mean \pm standard deviation (SD). The student's $t$-test was employed to assess statistical significant difference of the results. Difference was considered statistically significant at $P<0.05$.

\section{Results and Discussion}

3.1. Surface Characterization of RGDS-Immobilized QDs. The surface modification of CTNPs with RGDS peptide was confirmed by FTIR as displayed in Figure 3. In the case of CTNPs spectrum, the introduction of mercaptoisobutyric on the surface of CTNPs was confirmed by the characteristic peak at 1700 and $3500 \mathrm{~cm}^{-1}$ as shown in Figure 3(a), which attributed to free carboxyl $(-\mathrm{COOH})$ and hydroxyl $(-\mathrm{OH})$ groups [27, 28]. Again, after reaction of CTNPs with RGDS, two new peaks at positions around $1648 \mathrm{~cm}^{-1}$ and $1540 \mathrm{~cm}^{-1}$ were observed in the spectrum of RCTNPs (Figure 3(b)) which are assigned to amide I (-CO-NH-) and amide II (CO-NH-) bonds, respectively [29]. After the reaction of free amine groups of CTNPs with RGDS peptide, the absorption at $1648 \mathrm{~cm}^{-1}$ and $1540 \mathrm{~cm}^{-1}$ appeared based on amide groups of CTNPs, indicating successful immobilization of RGDS peptide on the surface of CTNPs [30].

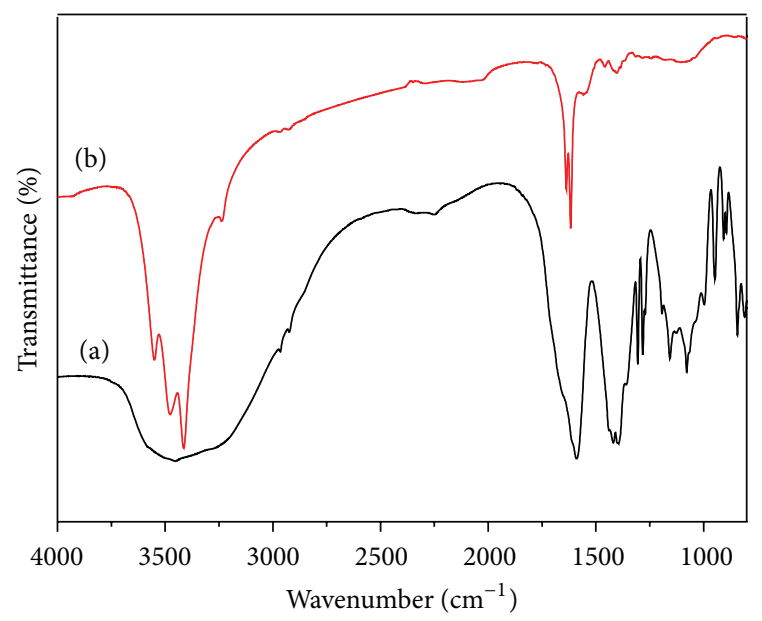

FIGURE 3: FT-IR patterns of CTNP (a) and RCTNP (b).

The TEM images of CTNPs and RCTNPs are shown in Figures 4(a) and 4(b), respectively. It is observed that CTNPs have a spherical morphology with an average diameter of ca. $4.1 \mathrm{~nm}$. Because of the small dimensions and high surface energy of the particles, it is easy for them to aggregate as seen in Figure 4(a). On the other hand, in case of RCTNPs the particles have a mean diameter of $5.1 \mathrm{~nm}$ are shaped spherically and comparatively low aggregated (Figure 4(b)). This larger particles size and nonaggregated particles morphology may have happened due to the conjugation of RGDS on the surface of CTNPs. Figure 5 shows the typical size and size distribution of synthesized CTNPs (Figure 5(a)) and RCTNPs (Figure 5(b)) measured by DLS. The average size of CTNPs as determined by DLS is ca. $37 \mathrm{~nm}$. On the other hand, average size of the RCTNPs is about $54 \mathrm{~nm}$. The size of the particles as determined by DLS is considerably larger than that determined by TEM. This is because the DLS technique gives a mean hydrodynamic diameter of CTNPs core surrounded by the organic and solvation layers, and this hydrodynamic diameter is influenced by the viscosity and the concentration of the solution. On the other hand, TEM gives the diameter of the core alone [31].

Synthesis of cadmium tellurite was also confirmed by UVvis absorption spectrum as shown in Figure 6. CTNPs sample showed an absorption onset at $491 \mathrm{~nm}$ and an emission at $521 \mathrm{~nm}$. The new peaks at 521 indicate that immobilized caused charge transfer of metal to ligand on CTNPs $[31,32]$.

3.2. Evaluation of Cytotoxicity. The status of the "live/dead" dye-stained fibroblasts containing CTNPs, RCTNPs, and control culture after 1 and 3 days of incubation is exhibited in Figure 7. The cytotoxic effect of CTNPs and RCTNPs on fibroblasts cells was analyzed by fluorescence microscopy using calcein-AM (green) and propidium iodide (red) as the marker which stain live and dead cells, respectively. Calcein-AM is highly lipophilic and cell membrane permeable. The calcein generated from the hydrolysis of calcein$\mathrm{AM}$ by cytosolic esterase in a viable cell emits strong green fluorescence. Therefore, calcein-AM only stains viable cells. 


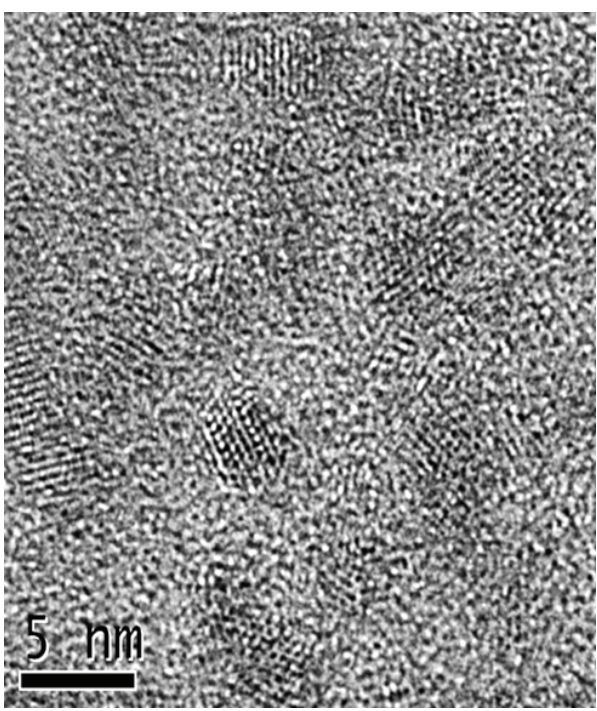

(a)

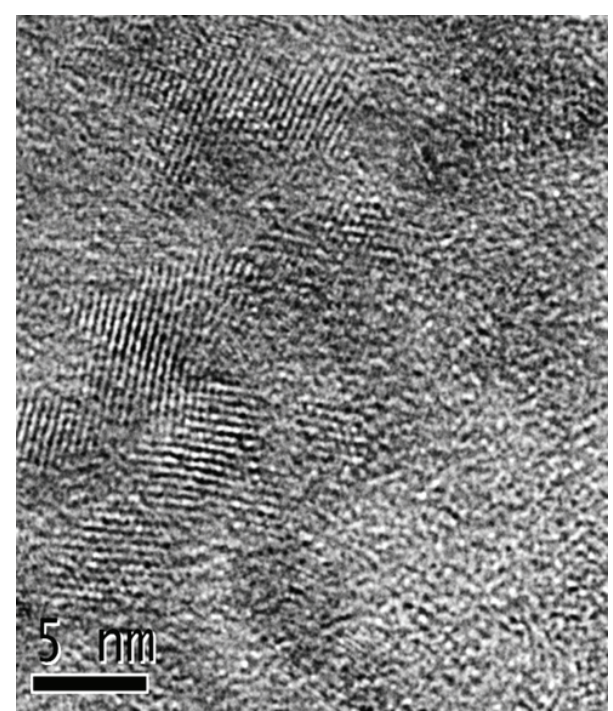

(b)

Figure 4: TEM images of CTNP (a) and RCTNP (b).

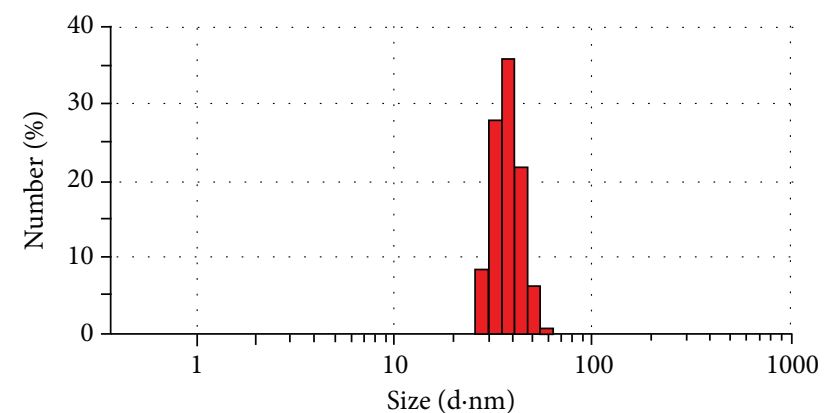

(a)

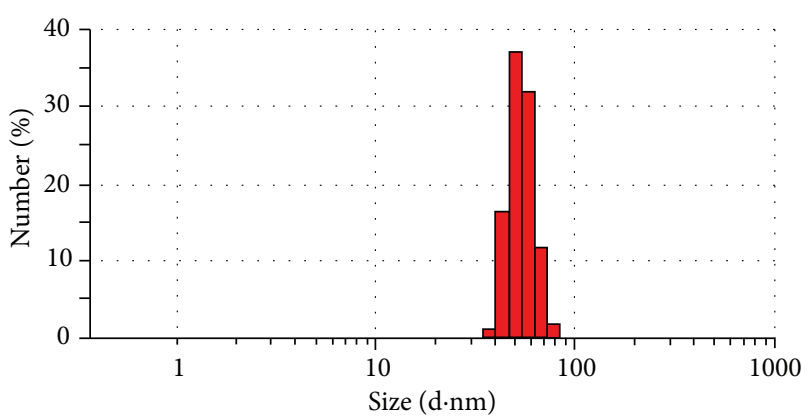

(b)

Figure 5: Particle size distribution of CTNP (a) and RCTNP (b) measured by dynamic light scattering (DLS).

In contrast, propidium iodide (PI), a nuclei staining dye, can pass through only the disordered areas of the dead cell membrane and intercalates with the DNA double helix of the cell to emit a red fluorescence (excitation: $535 \mathrm{~nm}$, emission: $617 \mathrm{~nm}$ ). By this qualitative method, the living and dead cells were, respectively, stained in green and red under fluorescence microscope. Figures 7 (a) and $7(d)$ indicates that the majority of the fibroblasts remained viable after 3

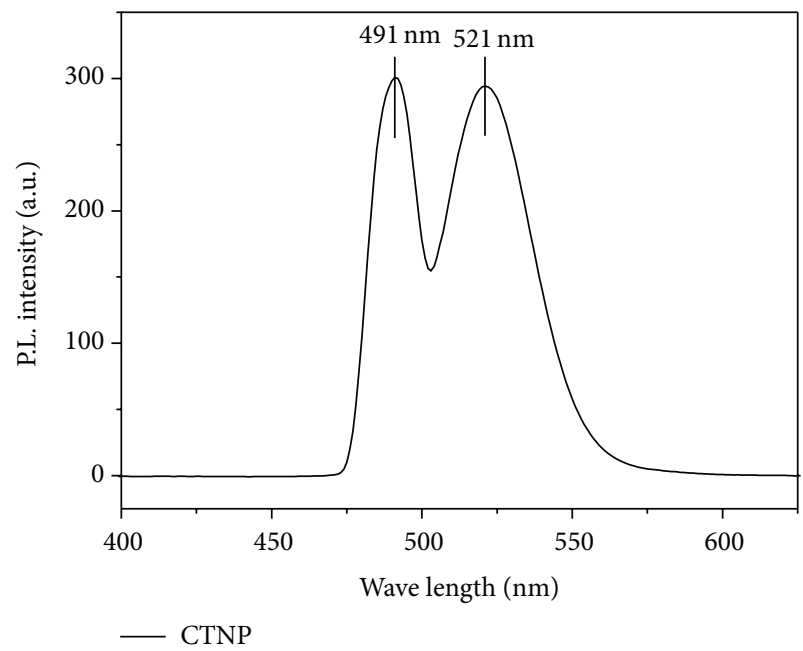

FIGURE 6: UV-vis absorption/emission spectra of mercaptoisobutyric acid-immobilized cadmium tellurite quantum dots (CTNP).

days of incubation irrespective of presence or absence of nanoparticles. However, after a culture of 3 days, some of the fibroblasts died in the case of CTNPs (Figures 7(b) and 7(e)). On the other hand, most of the fibroblasts remain viable in the presence of RCTNPs and control culture (Figures $7(\mathrm{a})$, $7(\mathrm{~d}), 7(\mathrm{c})$, and $7(\mathrm{f}))$, though a nonsignificant number of cells became dead in each of these cases. Possible explanation of this large decrease in cell viability in case of CTNPs is that (i) toxic cadmium ion $\left(\mathrm{Cd}^{2+}\right)$ releases from CTNPs due to surface oxidation and causes the cells death $[8,30]$; (ii) formation of reactive oxygen spices (ROS) and ROS reacts with cellular biomolecules, resulting in their damage, degradation, and finally loss of function [33]; (ii) nanoparticles are taken up by the cells as a result of endocytosis, which results in disruption of cell membrane; or (iii) weak cell adhesive 


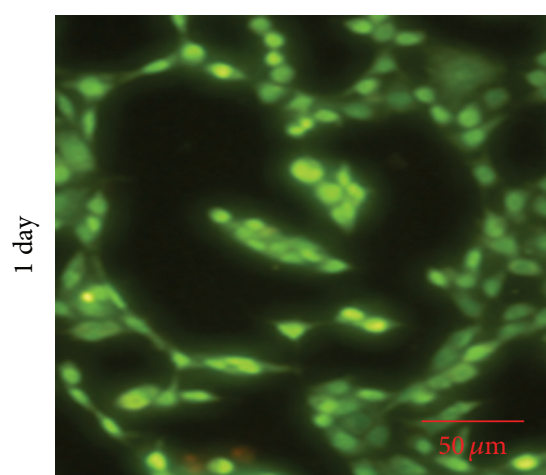

(a)

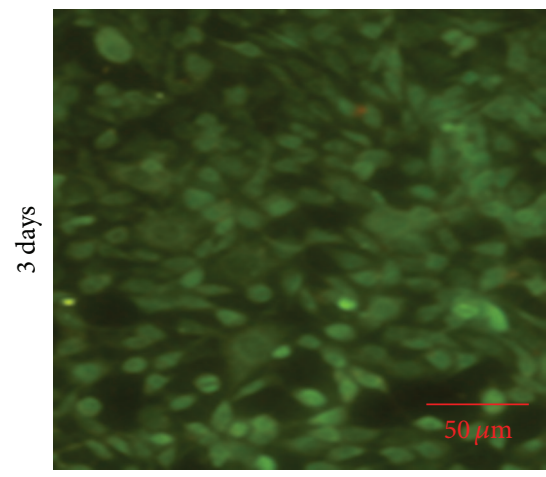

(d)

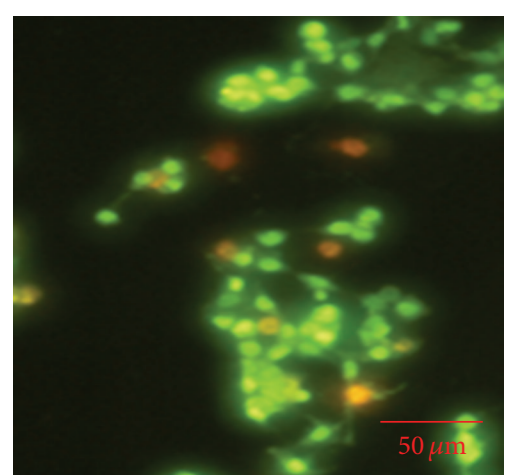

(b)

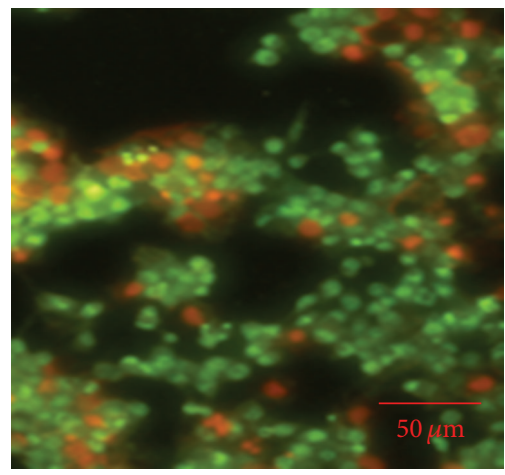

(e)

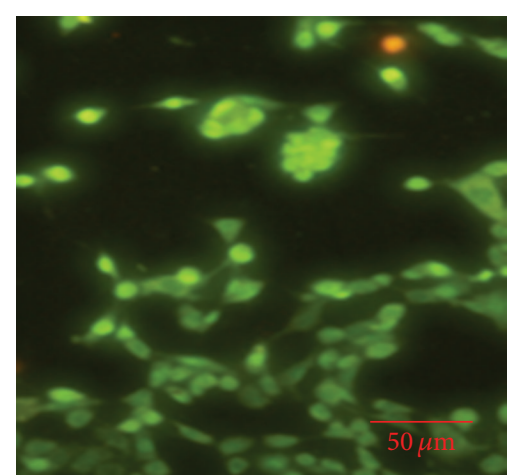

(c)

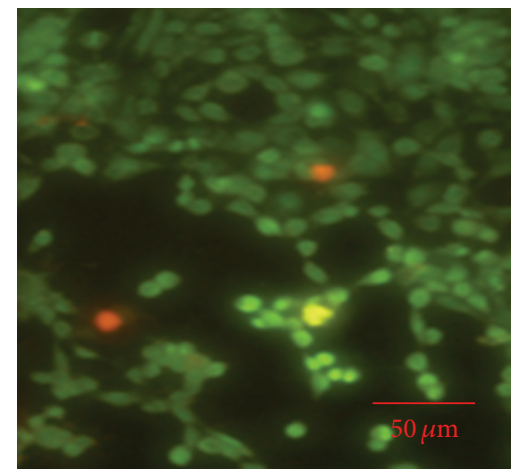

(f)

FIGURE 7: Fluorescence microscope image of live/dead dye-stained fibroblasts cultured for 1 and 3 days in a polystyrene culture dish ((a), (d)) and in the presence of CTNP ((b), (e)) and RCTNP ((c), (f)). Live and dead cells were stained and visualized in green and red, respectively, under a fluorescence microscope.

interaction with CTNPs promotes apoptosis (programmed cell death). Because mercaptoisobutyric acid is the least solubilizing ligand. It alone cannot protect CTNPs from surface oxidation and diffusion of $\mathrm{Cd}^{2+}$ ions from CTNPs for a longer period. It is unable to make CTNPs biological inert. Therefore, most of the cells died in the presence of CTNPs $[8,34]$. Again, cell viability of RCTNPs is moderately high due to the well-protected surface coating by biocompatible RGDS peptide which reduces the release of cadmium ion $\left(\mathrm{Cd}^{2+}\right)$ and formation of ROS. On the other hand, RCTNPs revealed almost no significant cytotoxic effect to cells up to 3 days. The increased cell viability can be explained by nutrient effect [34]. Besides, the low toxicity of nanoparticles derived with RGDS peptide may be attributed to the fact that these ligands act as cellular markers that are targeted at the surface receptors expressed on the cell surface without being internalized. Receptors are highly regulated cell surface proteins which mediate specific interactions between the cells and their extracellular milieu, and they are generally localized on the plasma membrane [34]. This result indicates that the cytotoxicity of CTNPs was improved by the conjugation of RGDS peptide on CTNPs (RCTNPs).

Viability of the cells cultured for 1,2 , and 3 days in the presence of CTNPs and RCTNPs was evaluated by MTT assay and represented in Figure 8. From the very first day of culture, the proliferation rate with RCTNPs was higher than that with CTNPs in standard deviation. However, after a culture of 2 and 3 days, cell proliferation rate with RCTNPs increased significantly $(P<0.05)$ compared to that with CTNPs. In other words, it can be stated that the surface modification of cadmium sulfide with RGDS could lessen the cytotoxicity of QDs considerably in comparison to that modified by mercaptobutyric acid.

3.3. Evaluation of Intracellular Uptake. The uptake of QDs nanoparticles to fibroblast (NIH 3T3) cells was evaluated by ICP, and the results were given in Figure 9. After oiligopeptide modifications, the nanoparticles uptaken by fibroblast cells increased greatly in comparison to unmodified nanoparticles and reached $2.5 \mathrm{pg} / \mathrm{cell}$ within the first day of culture, much higher than that by unmodified nanoparticles [35]. The maximum uptake of RCTNPs appeared at $9.7 \mathrm{pg} / \mathrm{cell}$ after 3 days of culture. The reason for the uptake improvement might be due to both conjugation of cell adhesion peptide and high solubility of RGTNPs in the cell membrane [32]. Furthermore, the higher uptake of RGDS-modified QDs indicates that the modification not only facilitated the nanoparticles to target specific cells, but also increased the yield of cell internalization. Similar results obtained from CMCS-SPIONs were reported by Shi et al. [35] in their results; the amount of nanoparticles internalized in the hMDCs is much more than that of SPIONs as determined by ICP-MS. The interaction between RGDS and integrin receptor expressed on the membrane of fibroblast might have contributed to 


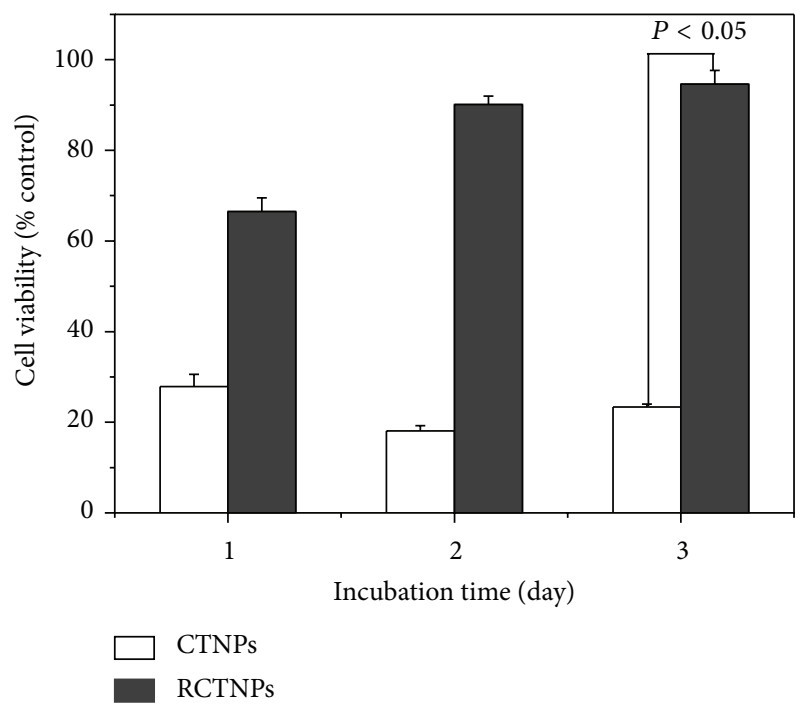

FIGURE 8: MTT assay, absorbance as a measure of cell proliferation from fibroblast cell cultured in the presence of CTNPs (the white bar) and RCTNPs (the black bar) for different time.

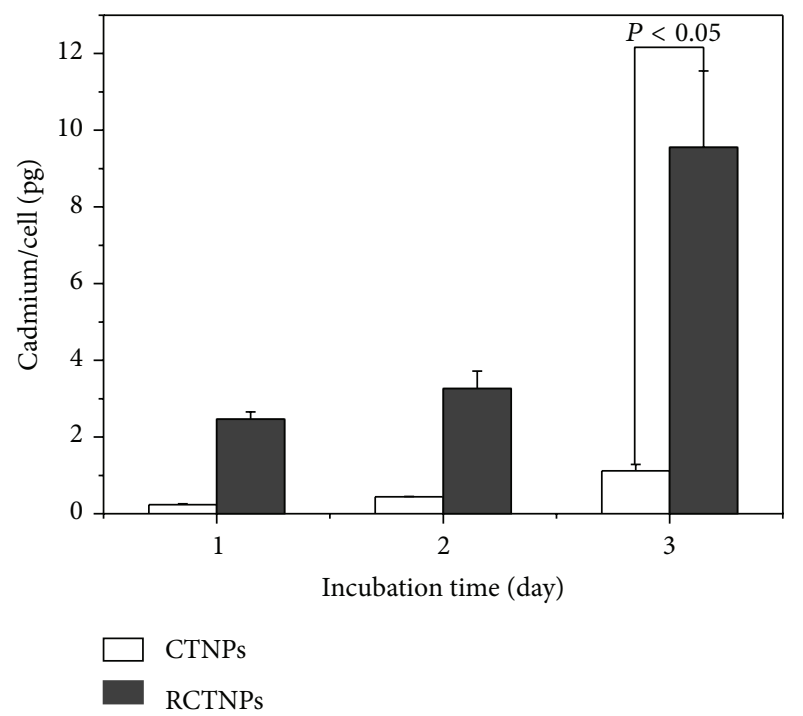

FIgURE 9: Amount of CTNPs (the white bar) and RCTNPs (the black bar) uptaken by fibroblasts at different incubation time as determined by ICP.

the improvement of quantum dots uptake based on receptor mediated endocytosis [30].

\section{Conclusions}

Mercaptoisobutyric acid-coated cadmium tellurite nanoparticles (CTNPs) were successfully conjugated with RGDS peptide. The oligo peptide-conjugated CTNPs (RCTNPs) showed considerably low cytotoxicity compared to the CTNPs. The results, from the experiment of intracellular uptake of the modified nanoparticles by ICP, showed that the RCTNPs can specifically recognize fibroblasts and demonstrated a higher efficiency of intracellular uptake into fibroblasts compared to the CTNPs, indicating an integrin-mediated specific interaction. These results suggest that the RCTNPs have a potential to be used in biological applications.

\section{Acknowledgment}

This research was supported by Basic Research Laboratory program (no. 2011-0020264) from the Ministry of Education, Science and Technology of Korea.

\section{References}

[1] J. Pan, Y. Wang, and S. S. Feng, "Formulation, characterization, and in vitro evaluation of quantum dots loaded in poly(Lactide)-Vitamin E TPGS nanoparticles for cellular and molecular imaging," Biotechnology and Bioengineering, vol. 101, no. 3, pp. 622-633, 2008.

[2] F. Pinaud, X. Michalet, L. A. Bentolila et al., "Advances in fluorescence imaging with quantum dot bio-probes," Biomaterials, vol. 27, no. 9, pp. 1679-1687, 2006.

[3] J. Pan and S. S. Feng, "Targeting and imaging cancer cells by Folate-decorated, quantum dots (QDs)-loaded nanoparticles of biodegradable polymers," Biomaterials, vol. 30, no. 6, pp. 11761183, 2009.

[4] K. S. Soppimath, T. M. Aminabhavi, A. R. Kulkarni, and W. E. Rudzinski, "Biodegradable polymeric nanoparticles as drug delivery devices," Journal of Controlled Release, vol. 70, no. 1-2, pp. 1-20, 2001.

[5] T. Jamieson, R. Bakhshi, D. Petrova, R. Pocock, M. Imani, and A. M. Seifalian, "Biological applications of quantum dots," Biomaterials, vol. 28, no. 31, pp. 4717-4732, 2007.

[6] A. P. Alivisatos, W. Gu, and C. Larabell, "Quantum dots as cellular probes," Annual Review of Biomedical Engineering, vol. 7, pp. 55-76, 2005.

[7] X. Michalet, F. F. Pinaud, L. A. Bentolila et al., "Quantum dots for live cells, in vivo imaging, and diagnostics," Science, vol. 307, no. 5709, pp. 538-544, 2005.

[8] A. M. Derfus, W. C. W. Chan, and S. N. Bhatia, "Probing the cytotoxicity of semiconductor quantum dots," Nano Letters, vol. 4, no. 1, pp. 11-18, 2004.

[9] E. T. Ben-Ari, "Nanoscale quantum dots hold promise for cancer applications," Journal of the National Cancer Institute, vol. 95, no. 7, pp. 502-504, 2003.

[10] H. Mattoussi, J. Matthew Mauro, E. R. Goldman et al., "Selfassembly of CdSe-ZnS quantum dot bioconjugates using an engineered recombinant protein," Journal of the American Chemical Society, vol. 122, no. 49, pp. 12142-12150, 2000.

[11] M. T. Fernández-Arguelles, A. Yakovlev, R. A. Sperling et al., "Synthesis and characterization of polymer-coated quantum dots with integrated acceptor dyes as FRET-based nanoprobes," Nano Letters, vol. 7, no. 9, pp. 2613-2617, 2007.

[12] Z. Chen, H. Chen, H. Meng et al., "Bio-distribution and metabolic paths of silica coated CdSeS quantum dots," Toxicology and Applied Pharmacology, vol. 230, no. 3, pp. 364-371, 2008.

[13] J. G. Rouse, C. M. Haslauer, E. G. Loboa, and N. A. MonteiroRiviere, "Cyclic tensile strain increases interactions between human epidermal keratinocytes and quantum dot nanoparticles," Toxicology In Vitro, vol. 22, no. 2, pp. 491-497, 2008.

[14] K. I. Hanaki, A. Momo, T. Oku et al., "Semiconductor quantum dot/albumin complex is a long-life and highly photostable 
endosome marker," Biochemical and Biophysical Research Communications, vol. 302, no. 3, pp. 496-501, 2003.

[15] H. M. Chen, X. F. Huang, L. Xu, J. Xu, K. J. Chen, and D. Feng, "Surface-defect-states photoluminescence in CdS nanocrystals prepared by one-step aqueous synthesis method," Superletter Microstructure, vol. 27, no. 3, pp. 1-5, 2000.

[16] X. Gao, W. C. W. Chan, and S. Nie, "Quantum-dot nanocrystals for ultrasensitive biological labeling and multicolor optical encoding," Journal of Biomedical Optics, vol. 7, no. 4, pp. 532537, 2002.

[17] C. C. Chen, C. P. Yet, H. N. Wang, and C. Y. Chao, "Self-assembly of monolayers of cadmium selenide nanocrystals with dual color emission," Langmuir, vol. 15, no. 20, pp. 6845-6850, 1999.

[18] F. Chen and D. Gerion, "Fluorescent CdSe/ZnS nanocrystalpeptide conjugates for long-term, nontoxic imaging and nuclear targeting in living cells," Nano Letters, vol. 4, no. 10, pp. 18271832, 2004.

[19] M. Bruchez, M. Moronne, P. Gin, S. Weiss, and A. P. Alivisatos, "Semiconductor nanocrystals as fluorescent biological labels," Science, vol. 281, no. 5385, pp. 2013-2016, 1998.

[20] W. C. W. Chan, D. J. Maxwell, X. Gao, R. E. Bailey, M. Han, and S. Nie, "Luminescent quantum dots for multiplexed biological detection and imaging," Current Opinion in Biotechnology, vol. 13, no. 1, pp. 40-46, 2002.

[21] F. Pinaud, D. King, H. P. Moore, and S. Weiss, "Bioactivation and Cell Targeting of Semiconductor CdSe/ZnS Nanocrystals with Phytochelatin-Related Peptides," Journal of the American Chemical Society, vol. 126, no. 19, pp. 6115-6123, 2004.

[22] W. C. W. Chan and S. Nie, "Quantum dot bioconjugates for ultrasensitive nonisotopic detection," Science, vol. 281, no. 5385, pp. 2016-2018, 1998.

[23] E. R. Goldman, G. P. Anderson, P. T. Tran, H. Mattoussi, P. T. Charles, and J. M. Mauro, "Conjugation of luminescent quantum dots with antibodies using an engineered adaptor protein to provide new reagents for fluoroimmunoassays," Analytical Chemistry, vol. 74, no. 4, pp. 841-847, 2002.

[24] D. Gerion, W. J. Parak, S. C. Williams, D. Zanchet, C. M. Micheel, and A. P. Alivisatos, "Sorting fluorescent nanocrystals with DNA," Journal of the American Chemical Society, vol. 124, no. 24, pp. 7070-7074, 2002.

[25] Z. C. Xing, M. J. Park, S. J. Han, M. J. Choi, B. H. Lee, and I. K. Kang, "Intracellular uptake of magnetite nanoparticles conjugated with RGDS-peptide," Macromolecular Research, vol. 19, no. 9, pp. 897-903, 2011.

[26] H. He, M. Feng, J. Hu et al., "Designed short RGD peptides for one-pot aqueous synthesis of integrin-binding CdTe and CdZnTe quantum dots," Applied Materials and Interfaces, vol. 4, no. 11, pp. 6362-6370, 2012.

[27] Z. Yang, X. Yang, and Z. Xu, "Molecular dynamics simulation of the melting behavior of Pt-Au nanoparticles with core-shell structure," Journal of Physical Chemistry C, vol. 112, no. 13, pp. 4937-4947, 2008.

[28] I. K. Kang, B. K. Kwon, J. H. Lee, and H. B. Lee, "Immobilization of proteins on poly(methyl methacrylate) films," Biomaterials, vol. 14, no. 10, pp. 787-792, 1993.

[29] K. M. Kamruzzaman Selim, Y. S. Ha, S. J. Kim et al., "Surface modification of magnetite nanoparticles using lactobionic acid and their interaction with hepatocytes," Biomaterials, vol. 28, no. 4, pp. 710-716, 2007.

[30] K. Von Der Mark, J. Park, S. Bauer, and P. Schmuki, "Nanoscale engineering of biomimetic surfaces: cues from the extracellular matrix," Cell and Tissue Research, vol. 339, no. 1, pp. 131-153, 2010.

[31] K. M. Kamruzzaman Selim, Z. C. Xing, H. Guo, and I. K. Kang, "Immobilization of lactobionic acid on the surface of cadmium sulfide nanoparticles and their interaction with hepatocytes," Journal of Materials Science: Materials in Medicine, vol. 20, no. 9, pp. 1945-1953, 2009.

[32] Y. Zhang, N. Kohler, and M. Zhang, "Surface modification of superparamagnetic magnetite nanoparticles and their intracellular uptake," Biomaterials, vol. 23, no. 7, pp. 1553-1561, 2002.

[33] K. M. Kamuruzzaman Selim, Z. C. Xing, M. J. Choi, Y. Chang, H. Guo, and I. K. Kang, "Reduced cytotoxicity of Insulinimmobilized CdS quantum dots using PEG as a spacer," Nanoscale Research Letters, vol. 6, no. 1, p. 528, 2011.

[34] A. K. Gupta and M. Gupta, "Cytotoxicity suppression and cellular uptake enhancement of surface modified magnetic nanoparticles," Biomaterials, vol. 26, no. 13, pp. 1565-1573, 2005.

[35] Z. Shi, K. G. Neoh, E. T. Kang et al., "(Carboxymethyl)chitosanmodified superparamagnetic iron oxide nanoparticles for magnetic resonance imaging of stem cells," Applied Materials \& Interfaces, vol. 1, no. 2, pp. 328-335, 2009. 

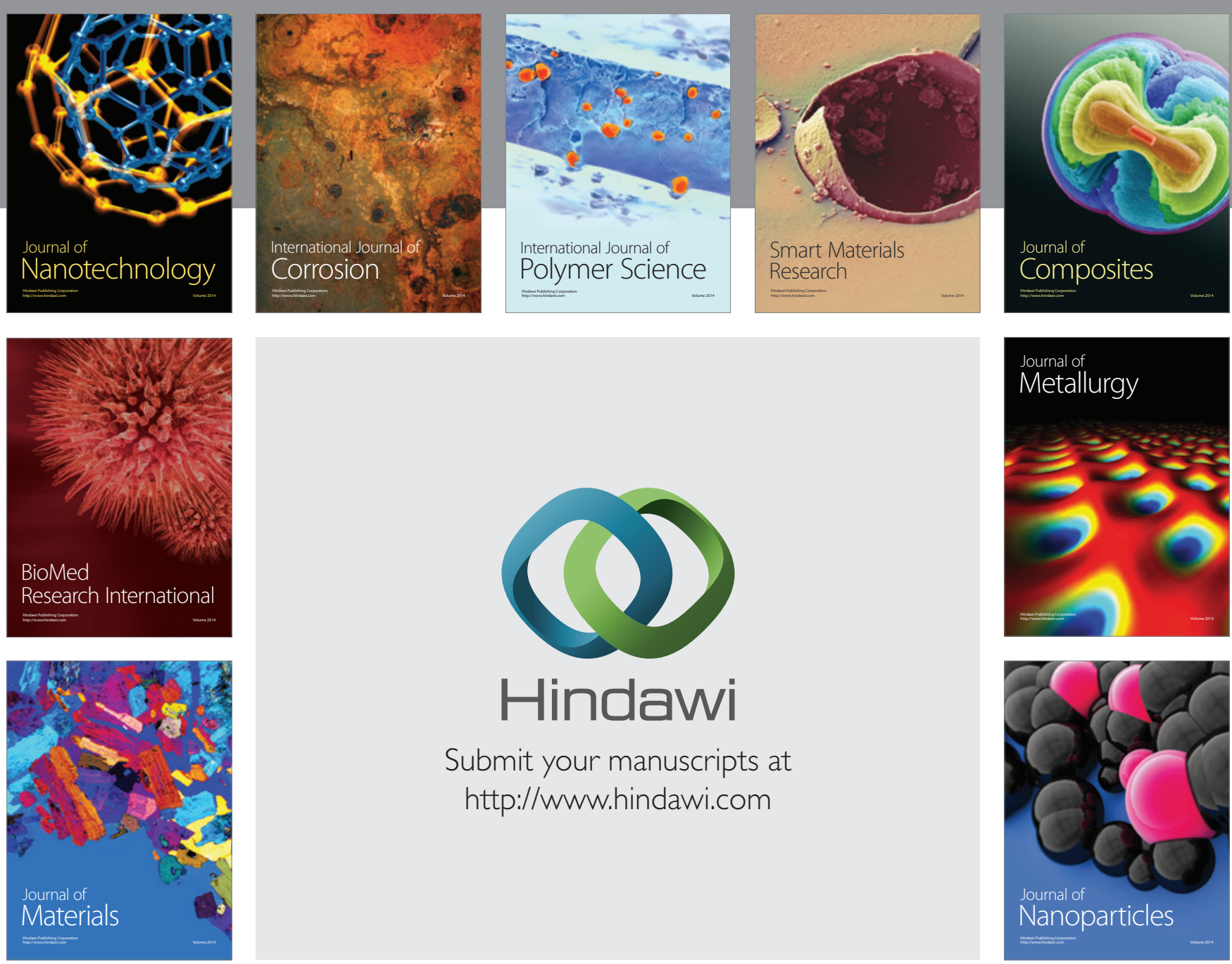

Submit your manuscripts at http://www.hindawi.com
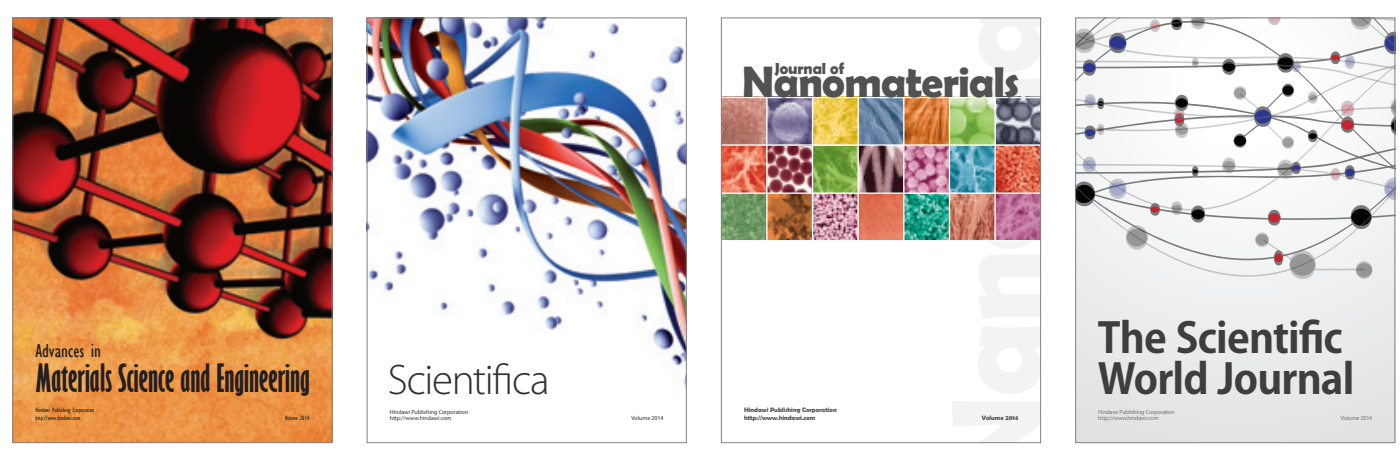

\section{The Scientific World Journal}
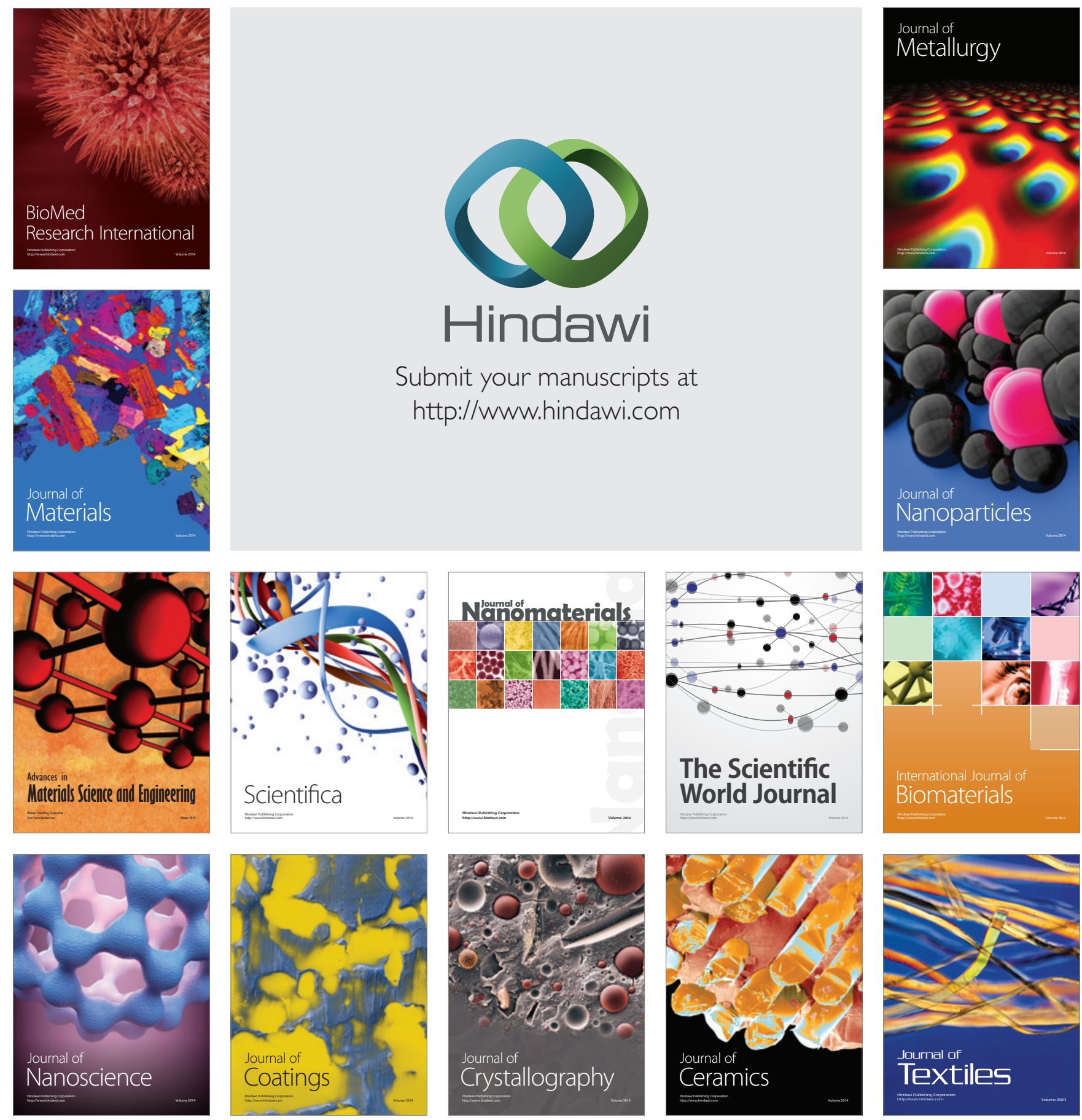